\title{
QUALIDADE DE VIDA E VOZ PÓS-RADIOTERAPIA: REPERCUSSÕES PARA A FONOAUDIOLOGIA
}

\author{
Quality of life and voice after radiotherapy: \\ Speech therapy repercussions
}

\author{
Renata Jacob Daniel Salomão de Campos ${ }^{(1)}$, Isabel Cristina Gonçalves Leite ${ }^{(2)}$
}

\begin{abstract}
RESUMO
Tema: o tratamento radioterápico do câncer de cabeça e pescoço tem mostrado resultados importantes no controle da doença quando neoadjuvante ou mesmo concomitante à quimioterapia. Pesquisas apontam um aumento da sobrevida após 5 anos de tratamento do câncer de laringe, o que torna necessário e justifica os esforços realizados na reabilitação da comunicação dos pacientes, e destaca a presença do profissional de Fonoaudiologia na equipe de radioterapia. Objetivo: descrever as seqüelas com repercussão fonoaudiológica, tais como alterações na qualidade vocal e na qualidade de vida, associadas ao tratamento proposto para tumores de cabeça e pescoço, em especial aquelas originadas após a radioterapia. Conclusão: Várias seqüelas, agudas e crônicas, como rouquidão, odinofagia e afonia, que envolvem dificuldades na comunicação oral e/ou deglutição dos pacientes após o tratamento radioterápico em cabeça e pescoço, interfere de maneira direta na comunicação, deglutição e qualidade de vida dos pacientes. Os profissionais envolvidos no tratamento desse câncer, dentre eles o fonoaudiólogo, através do conhecimento sobre o tratamento e as fragilidades terapêuticas que comprometem a qualidade vocal e qualidade de vida desses pacientes, busca contribuir no processo de recuperação minimizando os danos causados pela doença e melhorando a condição de saúde do indivíduo.
\end{abstract}

DESCRITORES: Radioterapia; Epidemiologia; Voz; Neoplasias de Cabeça e Pescoço; Qualidade de vida

\section{INTRODUÇÃO}

O câncer é resultante de um processo multifatorial que consiste em diversos estágios, caracterizando uma doença que requer o mais alto nível técnico de tratamento realizado por diversos profissionais da área da saúde. Neste contexto, a fonoaudiologia se insere ${ }^{1}$.

A literatura internacional aponta as neoplasias como segunda causa mais importante de morte, apenas superada pelas doenças cardiovasculares

(1) Fonoaudióloga Clínica do Instituto Oncológico de Juiz de Fora; Especialista em Voz. Mestre em Educação; Mestranda em Saúde Brasileira pela Universidade Federal de Juiz de Fora.

(2) Cirurgiã Dentista; Professora Adjunta da Universidade Federal de Juiz de Fora; Mestre e Doutora em Saúde Pública pela Escola Nacional de Saúde Pública-FIOCRUZ.

Conflito de interesses: inexistente nas populações feminina e masculina com mais de 40 anos $^{2}$.

Particularmente no caso dos cânceres de cabeça e pescoço, o tratamento proposto, habitualmente, envolve cirurgia, radioterapia, quimioterapia ou uma combinação dos mesmos. Portanto, cirurgiões, radioterapeutas e oncologistas devem participar dos tratamentos para contribuírem no melhor controle loco-regional da doença, e simultaneamente otimizar a sobrevida após a cura por meio da preservação da função de comunicação do paciente. Entretanto, embora a cura possa se alcançada em $60 \%$ dos casos, esta não significa necessariamente melhora da qualidade de vida dos mesmos, permanecendo este como o grande desafio da oncologia, em especial as funções relativas à reintegração social dos afetados, em especial a fala ${ }^{3}$.

A intervenção fonoaudiológica é comum e importante para os pacientes que foram submetidos 
à cirurgia de cabeça e pescoço ${ }^{4}$. $\mathrm{O}$ acompanhamento é feito desde a fase pré-cirúrgica, fundamentado na orientação sobre a reabilitação proposta após o procedimento cirúrgico. Neste momento, algumas medidas vocais e da deglutição são aferidas para serem comparadas no pós-operatório, com objetivo de minimizar as alterações da comunicação presentes após o tratamento do câncer.

A literatura científica ${ }^{4}$ refere-se à intervenção fonoaudiológica no pré e pós-operatórios de cabeça e pescoço, contudo poucas referências descrevem a importância deste profissional nas equipes de radioterapia e quimioterapia. A reabilitação oncológica se faz necessária também junto aos pacientes que foram submetidos à radiação, pois são descritas seqüelas presentes após estes tratamentos, que envolvem dificuldades na comunicação oral e/ ou deglutição desses pacientes.

Nesse sentido o presente artigo propõe por meio de uma revisão da literatura descrever as seqüelas com repercussão fonoaudiológica, tais como alterações na qualidade vocal e de vida, associadas ao tratamento proposto para tumores de cabeça e pescoço, em especial aquelas originadas após a radioterapia.

\section{MÉTODOS}

Os trabalhos utilizados foram selecionados por meio de pesquisa bibliográfica dos textos indexados nas seguintes bases bibliográficas dados: Medline; Scielo (Scientific Eletronic Library Online); Pubmed; Periódicos CAPES. Também foram utilizados livros da área, monografias, dissertações de mestrado e teses de doutorados.

Esta revisão de literatura teve origem no projeto de pesquisa desenvolvido pelos autores e que atendeu aos requisitos da Declaração de Helsinque e às recomendações da Resolução 196/96 do Conselho Nacional de Saúde, tendo sido aprovado pelo Comitê de Ética em Pesquisas em Seres Humanos da Propesq/ UFJF sob parecer n. 236/2007.

Foram utilizadas palavras-chaves para a busca nos periódicos nas línguas portuguesa e inglesa, sendo estas: voz; desordens vocais; radioterapia; qualidade vocal; qualidade de vida; alterações após radioterapia; fonoaudiologia e radioterapia. Estes dados foram selecionados inicialmente, separados e posteriormente foram cruzados para melhor seleção dos artigos. Procurou-se destacar os estudos mais recentes, ou seja, de 2000 a 2009 e alguns artigos de referência da década de 1990 também contribuíram para a revisão.

\section{REVISÃO DA LITERATURA}

\section{Câncer de cabeça e pescoço: aspectos epidemiológicos}

Conforme apontam os estudos epidemiológicos, o aumento da expectativa de vida associa-se à tendência de crescimento da incidência de câncer de cabeça e pescoço em todo o mundo. Além dos fatores genéticos, a exposição ao tabaco, especialmente associado ao álcool, exibe comportamento de potente variável que aumenta as chances de ocorrência deste tumor ${ }^{5,6}$.

Os carcinomas de cabeça e pescoço representam $18 \%$ de todos os tumores malignos aproximadamente. Cerca de 90\% destas neoplasias são manifestadas sob forma de carcinomas espinocelulares, e em menor freqüência os de natureza neuroendócrina, originados de glândulas salivares menores, sarcomas e linfomas ${ }^{4}$.

O câncer de boca e orofaringe tem comportamento agressivo, sendo a incidência no Brasil uma das mais altas no mundo. Segundo Instituto Nacional do Câncer e Ministério da Saúde ${ }^{2}$, os registros hospitalares revelam que o câncer de cavidade oral está em oitavo lugar como o câncer mais freqüente nos homens e nono lugar nas mulheres, por cem mil habitantes, no estado de Minas Gerais. É considerado o câncer mais comum da região da cabeça e pescoço, excluindo-se o câncer de pele não-melanoma. A estimativa foi de 466.730 novos casos de câncer no Brasil em 2008, sendo que o de cavidade oral deveria reunir um total de 14.160. Destes, 8.010 casos ocorreram na região sudeste brasileira.

Os tumores da faringe estão preferencialmente localizados na hipofaringe e apresentam o mesmo padrão histopatológico e epidemiológico dos tumores de cabeça e pescoço ${ }^{4}$.

O câncer de laringe é considerado um dos mais comuns dentre os tumores da região da cabeça e pescoço, correspondendo a cerca de $25 \%$ dos tumores malignos desta área e $2 \%$ de todas as doenças malignas. A literatura afirma que a preservação da voz é um importante parâmetro na decisão do tipo de tratamento, surgindo os protocolos de preservação de órgãos nos quais se preconiza a radioterapia e/ou quimioterapia ${ }^{4}$.

\section{Tratamento com preservação de órgãos: radioterapia e/ou quimioterapia em cabeça e pescoço}

Estudos mostram bons resultados no tratamento da doença pela preservação do órgão laríngeo através de tratamentos combinados de radioterapia e quimioterapia, não comprometendo os resultados em termos de tempo de sobrevida ${ }^{2}$. Porém, nem 
sempre a preservação da laringe se torna um tratamento que resguarde realmente todas as funções. Os pacientes possivelmente apresentam alterações de ordem específicas na comunicação e deglutição.

A terapia de radiação tem sido usada há mais de 100 anos. É um método comprovado para controlar malignidades, que possibilita o aumento da sobrevida de indivíduos acometidos por câncer. É usada em mais da metade de todos os tratamentos de câncer e, em alguns casos, é o tratamento de eleição, prevalecendo sobre todas as escolhas terapêuticas. Em outros casos, é usada em combinação com quimioterapia e/ou cirurgia. Apesar dos benefícios desta terapia, apresenta efeitos colaterais como todos os tratamentos contra doenças ${ }^{7}$.

O tratamento radioterápico no câncer de cabeça e pescoço tem o objetivo de liberar a dose adequada para o controle do tumor, preservando tecidos vizinhos sadios. A dosagem mínima é de 65Gy para controle local desta doença ${ }^{4}$. Os tumores iniciais (T1 e T2) podem ser tratados com alto grau de sucesso pela cirurgia e pela radioterapia, porém as lesões superficiais e exofíticas são mais radiossensíveis, enquanto as lesões infiltrativas, ulceradas e hipóxicas respondem melhor à conduta cirúrgica.

Segundo parâmetros estabelecidos pelo Instituto Nacional do Câncer e o Ministério da Saúde ${ }^{8}$ devem ser aplicados 6.600 cGy em lesões iniciais de cabeça e pescoço (T1ou T2). Nas lesões avançadas (T3 e T4), aplica-se 7.000cGy em lesão primária. O esvaziamento cervical radical ou radical modificado é indicado, dependendo do estádio da doença cervical, bem como em casos de metástase cervical residual ou de recidiva cervical.

\section{Seqüelas identificadas nos pacientes após radioterapia em cabeça e pescoço}

O tratamento radioterápico para pacientes acometidos de tumores malignos de cabeça e pescoço exibe complicações orais importantes, dentre elas mucosite, xerostomia, cáries, perda do paladar, infecções secundárias, osteorradionecrose e trismo ${ }^{9}$. Estes efeitos colaterais podem ser classificados em formas agudas, por exemplo: eritema, mucosite, edema laríngeo moderado e rouquidão temporária, ou crônicas, tais como necrose do tecido, função da saliva prejudicada, edemas e disfonia severa ${ }^{4,6}$. Estas manifestações crônicas foram descritas como complicações tardias da radioterapia, acrescentando-se outras manifestações fonoaudiológicas como fibrose laríngea e imobilidade bilateral das pregas vocais ${ }^{10}$.

Alterações no tecido laríngeo e/ou em torno do arcabouço laríngeo restringem o movimento necessário das cartilagens e músculos, sendo modificado o fechamento glótico para a produção vocal, causa esta relatada pelos pacientes como dificuldade de fonação e rouquidão.

\section{Avaliação fonoaudiológica vocal em pacientes oncológicos}

A fonoaudiologia tem parâmetros de medida subjetiva e objetiva da qualidade vocal, tornando importante o acompanhamento fonoaudiológico durante a radioterapia. Sua intervenção visa facilitar a produção vocal e também poderá trazer resultados importantes na qualidade de vida dos pacientes com tumores de cabeça e pescoço, visto a facilidade de manterem a comunicação oral e convívio social.

A avaliação subjetiva da voz é a avaliação clássica da qualidade vocal, ou seja, avaliação perceptiva-auditiva. E a avaliação objetiva é feita por um programa de voz computadorizado. Normalmente, armazena um arquivo vocal contendo uma vogal sustentada (vogal /a/ ou /e/) e fala encadeada seguida de fala espontânea, que serão analisadas por um especialista em voz. O profissional procura delimitar e correlacionar os parâmetros vocais encontrados em normais ou alterados e em qual proporção isto acontece. A escala japonesa GRBAS é usada internacionalmente, sendo um método simples de avaliar o grau global da disfonia (G), que é composto por 4 fatores independentes: rugosidade $(R)$, soprosidade $(S)$, astenia $(A)$ e tensão $(T)$, considerados fatores importantes na definição de uma voz disfônica. Vale ressaltar que os parâmetros de astenia e tensão são excludentes, por motivos óbvios. Recentemente foi proposto acrescentar o fator instabilidade (I) tornando escala GRBASI ${ }^{1}$.

A avaliação objetiva refere à avaliação acústica do sinal sonoro, que é medido através de uma amostra vocal, geralmente vogal sustentada, por um microfone acoplado ao computador que contenha o laboratório de voz. Esta amostra será analisada pelo software do clinico e será realizada a medida da freqüência fundamental. É importante ressaltar que a normatização do uso do laboratório de voz requer formação específica e experiência do fonoaudiólogo para análise dos dados encontrados no programa de voz ${ }^{1}$.

Na Figura 1 são apresentadas medidas subjetivas e objetivas da qualidade vocal utilizadas na prática clínica do fonoaudiólogo especializado.

\section{Reabilitação oncológica}

Em um estudo sobre os resultados funcionais do tratamento para câncer de laringe avançado, no qual foram avaliadas funções relativas à comunicação e deglutição, foram obtidos resultados claros que os pacientes com este tumor não desenvolvem bem a comunicação espontânea quando há remo- 


\begin{tabular}{|l|l|l|}
\hline PARÂMETROS & NORMAL & ALTERADO \\
\hline MEDIDAS & & \\
\hline SUBJETIVAS & G=0 & $\begin{array}{l}\mathrm{G}=1 ; 2 \text { ou } 3 \\
\text { R; B; S; I = } \\
1-\text { Discreto; } 2-\text { Moderado; } \\
3-\text { Severo }\end{array}$ \\
\hline & & Homem \# $113 \mathrm{~Hz}$ \\
OBJETIVAS & Homem $=113 \mathrm{~Hz}$ & $\begin{array}{l}\text { Mulher \# } 295 \mathrm{~Hz} \\
\text { Criança \# } 236 \mathrm{~Hz}\end{array}$ \\
\hline
\end{tabular}

$\mathrm{G}$ = grau global de disfonia; $\mathrm{R}$ = rouquidão; $\mathrm{B}$ = soprosidade $\mathrm{A}$ = astenia; $\mathrm{S}=$ tensão; I = irregularidade.

Fonte: Behlau, 2005

Figura 1 - Medidas subjetivas e objetivas da qualidade vocal

ção total do órgão laríngeo. No mesmo trabalho, os autores focalizaram os resultados da reabilitação de pacientes laringectomizados totais, concluindo que somente porcentagens relativamente pequenas de pacientes $(6 \%)$ desenvolveram a voz esofágica. Este achado ressaltou a importância de um tratamento que preserve o órgão com o objetivo de ser obter uma melhor reabilitação ${ }^{11}$.

Dentre as alterações vocais após radioterapia, os músculos expostos ao tratamento podem desenvolver fibrose e edema, contudo os efeitos gerados na qualidade vocal não são bem compreendidos. Estes achados evidenciam a necessidade de pesquisa na área e atendimentos fonoaudiológicos a estes pacientes, protocolando resultados obtidos, sejam por avaliação objetiva ou subjetiva.

Os pacientes que tiveram tumores localizados na região laríngea exibiram mais alterações vocais que os pacientes que apresentavam tumores em outras regiões da cabeça e pescoço.

Em estudo prospectivo, $80 \%$ dos pacientes que foram avaliados apresentaram alteração na qualidade vocal após 1 ano do tratamento de radioterapia ${ }^{12}$.

A inteligibilidade da fala após o tratamento radioterápico em cabeça e pescoço é pouco descrita ${ }^{11}$, porém há relato de autores que os resultados após esta modalidade de tratamento são relativamente bons, ou seja, a inteligibilidade de parte dos pacientes não é alterada ${ }^{13}$.

Um estudo avaliou as características da voz e do discurso dos pacientes que se submeteram a diferentes tratamentos para o câncer de laringe comparando com indivíduos laríngeos normais. Foram avaliados 22 pacientes do sexo masculino, sendo que destes, cinco receberam tratamento radioterápico, seis fizeram laringectomia parcial, seis foram submetidos a laringectomia total e cinco eram indivíduos laríngeos normais. O estudo concluiu que todos os pacientes desenvolveram ou mantiveram uma fonte fonatória após o tratamento e puderam usar o discurso funcionalmente, sendo que o grupo que foi submetido à radiação obteve as vozes mais próximas do grupo controle, sendo o achado oposto verdadeiro para os grupos cirúrgicos, especialmente para os laringectomizados totais ${ }^{3}$.

Em outro estudo, no qual foi mensurada a alteração vocal, foi concluído que, dos 15 pacientes submetidos ao tratamento de radioterapia e quimioterapia para câncer de laringe, 33\% (cinco pacientes) apresentaram qualidade vocal adequada ou disfonia discreta, $40 \%$ (seis pacientes) apresentaram disfonia moderada e $27 \%$ (quatro pacientes) apresentaram disfonia severa. As medidas vocais obtidas foram a análise perceptiva-auditiva e acústica da voz ${ }^{4}$.

Sobre qualidade vocal após tratamento radioterápico para tratamento do tumor glótico inicial, os resultados do estudo evidenciaram que $11 \%$ dos casos tinham vozes normais, $44 \%$ tinham disfonias discretas, 27 a $28 \%$ disfonia moderada e de 16 a $17 \%$ tinham disfonia severa. A forma de avaliação foi subjetiva, através da análise auditiva, e a avaliação objetiva, através da análise acústica da qualidade vocal ${ }^{12}$.

Em estudo sobre avaliação vocal e acústica de carcinoma epidermoide de seio piriforme após terapêutica com quimioterapia e radioterapia em que o método foi a aplicação da avaliação vocal, que consistiu em uma gravação vocal (vogal sustentada e texto), análise acústica objetiva (parâmetros 
temporal e de freqüência vocal) e uma avaliação perceptiva auditiva da qualidade da voz por meio da escala de GRBAS ${ }^{13}$, os autores identificaram que os parâmetros acústicos de instabilidade da freqüência fundamental e o tempo máximo de fonação eram diferentes quando comparados com indivíduos normais.

\section{Qualidade de vida em pacientes submetidos a tratamento oncológico em cabeça e pescoço}

A importância da qualidade de vida dos pacientes pós-tratamento de câncer de cabeça e pescoço tem sido um assunto pesquisado por fonoaudiólogos, visto a forte ligação da comunicação e a integração social, esta última, às vezes, ausente nestes pacientes, em virtude da desfiguração facial. Pesquisas trazem resultados importantes de alteração da qualidade de vida de pacientes submetidos a tratamentos diversos em cabeça e pescoço quando comparados com a população normal ${ }^{14}$.

A criação e validação do Vocal Handicap Index (VHI) - Índice de Desvantagem Vocal - (IDV) ocorreu em 1997 nos Estados Unidos da América, traduzido por Behlau et al no Brasil. ${ }^{15}$ Este instrumento visa detectar através do questionário quais são as conseqüências psico-sociais geradas pela alteração da qualidade vocal ${ }^{16}$

O índice de desvantagem vocal é sugerido para aplicação em pacientes com a finalidade de detectar a percepção em relação a sua disfonia, podendo ser um instrumento valioso para acompanhamento no tratamento por parte do paciente ${ }^{17}$. Houve a tradução para o português deste questionário que tem sido usado desde então em alguns serviços no país ${ }^{16}$.

Em uma instituição no Brasil foi avaliada a qualidade de vida a longo prazo de pacientes tratados por câncer de cabeça e pescoço ${ }^{18}$. Foi aplicado o protocolo da Universidade de Washington devidamente traduzido e o prejuízo foi maior na fala dos pacientes submetidos a tratamento de tumores de hipofaringe e laringe. Concluíram que este questionário é uma ferramenta efetiva para avaliar a qualidade de vida após o tratamento do câncer de cabeça e pescoço. Este estudo valida a importância da aplicação dos protocolos de qualidade de vida, visando verificar o índice de prejuízo em que o paciente se encontra, o encaminhando, assim, para a reabilitação vocal adequada.

As alterações vocais após o tratamento de radioterapia em 25 pacientes foram estudadas, sendo 10 localizados na laringe e 15 em outros sítios da cabeça e pescoço. Constatou-se que todas as medidas estavam mais comprometidas no grupo de tumores não laríngeos, bem como foi também maior o prejuízo nas medidas referidas do índice de desvantagem vocal ${ }^{19}$.

A terapia vocal é um método efetivo para os pacientes que tinham problemas de voz após o tratamento para carcinoma glótico inicial ${ }^{19} \mathrm{~A}$ melhoria foi significativa quando analisados os resultados do questionário de índice de desvantagem vocal e foi também confirmada através dos parâmetros vocais objetivos avaliados.

Pacientes portadores de tumores laríngeos mostraram uma deterioração da qualidade vocal correlacionado com o IDV expressivamente menor em estudo recente que analisou medidas objetivas produzidas pela escala GRBAS associando com a avaliação da qualidade de vida por meio do índice de desvantagem vocal (IDV) ${ }^{13}$. Com estes achados, os autores concluíram que são interessantes as avaliações da função vocal e da qualidade de vida após tratamento de radioterapia e quimioterapia, com objetivo de permitir a análise da conservação da função vocal e, eventualmente, propor um acompanhamento fonoterápico.

Em estudo comparativo sobre problemas de voz e qualidade de vida através do índice de desvantagem vocal (IDV) após radioterapia, cirurgia endoscópica e laser para carcinoma glótico inicial, conclui-se que uma porcentagem elevada dos pacientes relatou problemas da voz na vida diária, sendo que $58 \%$ dos pacientes submetidos à radioterapia obtiveram contagens anormais no IDV ${ }^{20}$. A partir destes achados, os autores concluíram que o tratamento para o câncer glótico inicial freqüentemente resulta em problemas da voz na vida diária, influenciando negativamente atividades sociais dos pacientes.

Em estudo sobre qualidade de vida após o tratamento de carcinoma laríngeo ${ }^{21}$, os autores concluíram que há piora significativa na qualidade de vida dos pacientes tanto entre aqueles que submetem ao tratamento cirúrgico, quanto naqueles com indicação para tratamento radioterapêutico; sendo que os que foram tratados com radioterapia parecem ter um maior impacto negativo na qualidade de vida, quando comparados com os pacientes que fizeram cirurgia.

A metodologia de avaliação de qualidade de vida de pacientes submetidos a tratamento para o câncer de cabeça e pescoço tem amadurecido muito nos últimos dez anos e que essa avaliação deve ser feita em pacientes tratados a curto e longo prazo. Os pacientes tratados após 18 meses tendem a estabilizarem nas respostas do protocolo de qualidade de vida ${ }^{22}$.

A necessidade do serviço de Fonoaudiologia é reforçada ao verificar estudo que mensurou através de uma escala de ansiedade e depressão 
aplicada a cônjuges e pacientes tratados para câncer de cabeça e pescoço ${ }^{23}$. Seus resultados indicam um percentual de $20 \%$ dos parceiros e de $27 \%$ dos pacientes com respostas positivas à escala de ansiedade e depressão. A porcentagem de respostas desfavoráveis dos pacientes foi relacionada à presença da sonda para alimentação, fala não bem expressiva, contatos sociais menores e um estilo passivo de lidar com atividades diárias e com as emoções. Este mesmo padrão de respostas entre os cônjuges foi relacionada à presença da sonda nasogástrica para alimentação de seus parceiros, um estilo de vitalidade menor e mais passivo e pouca interação às atividades da vida diária.

A atuação fonoaudiológica é de suma importância, visto que tanto o tumor de cabeça e pescoço, quanto o tratamento afetam aspectos da vida diária que resultam na piora da qualidade de vida. $\mathrm{O}$ acompanhamento fonoaudiológico na fase pré ou pós-radioterapia visa à preservação ou readaptação das funções comunicativas do indivíduo, o que traz claro impacto na qualidade de vida dos mesmos.

\section{CONCLUSÃO}

A presente revisão evidenciou várias seqüelas, agudas e crônicas, como rouquidão, odinofagia e afonia, que envolvem dificuldades na comunicação oral e/ou deglutição dos pacientes após o tratamento radioterápico em cabeça e pescoço. Essas condições interferem de maneira direta na qualidade de vida dos pacientes. Os profissionais envolvidos no tratamento desse câncer, dentre eles o fonoaudiólogo, por meio do conhecimento sobre o tratamento e as fragilidades terapêuticas que comprometem a qualidade vocal e de vida desses pacientes, devem direcionar esforços que visem contribuir no processo de recuperação minimizando os danos causados pela doença e melhorando a condição de saúde do indivíduo.

O acompanhamento fonoaudiológico dos pacientes submetidos ao tratamento, inclusive radio e/ou quimioterápico, é um procedimento importante e que deve ter como objetivo minimizar as seqüelas presentes que interferem na comunicação oral. As avaliações subjetiva e objetiva podem subsidiar o tratamento e também o feedback para o paciente de seu desempenho vocal.

São necessários novos estudos que comparem a qualidade de vida e voz dos pacientes submetidos ao tratamento fonoaudiológico e grupos controles, bem como construção/avaliação de protocolos de encaminhamento, intervenção e proservação dos casos provenientes dos ambulatórios de radio e quimioterapia.

\section{AGRADECIMENTOS}

Aos Diretores do Instituto Oncológico de Juiz de Fora, Dr. Narciso Francisco Pazzinato, Dra. Tereza Cristina Esteve e Dr. Olamir Rossini Júnior. À radioterapeuta Luciana Vignoli e às colegas Cristina Maciel Tostes Vieira, Dra. Elisabete Carrara-de Angelis e Dra. Sandra Pela. À coordenadora de ensino do Programa de Pós-graduação em Saúde Brasileira da Universidade Federal de Juiz de Fora, Dra. Darcília Maria Nagen da Costa.

\section{ABSTRACT \\ Background: radiotherapy for head and neck cancer has been important for disease control, both as neo-adjuvant and concomitant to chemotherapy. 5-year survival rates after larynx cancer have increased, justifying the efforts towards rehabilitation of patients' communication and highlighting the importance of the speech-language pathologist in the radiotherapy team. New scientific and therapeutic breakthroughs have turned organ preservation into a strong ally in head and neck cancer treatment. Purpose: to show the speech-language-related sequelae, such as changes in vocal and life quality, related to treatment for head and neck tumors, especially those arising after radiotherapy. Conclusion: many acute and chronic sequelae, such as hoarseness, odinophagy and aphony, involving difficulties in oral communication and/or swallowing in patients after neck and head radiotherapy treatment, interfering with communication, swallowing, and life quality of patients in a direct manner. The professionals involved with the treatment of this cancer, with the speech-language pathologist among them, through the knowledge on the treatment and its therapeutic weaknesses that jeopardize the vocal quality and life quality of those patients, seek to contribute in the recovery process in order to minimize damages caused by the disease and improving the individual's health condition.}

Keywords: Radiotherapy; Epidemiology; Voice; Head and Neck Neoplasms; Quality of life 


\section{REFERÊNCIAS}

1. Behlau M. Voz o livro do especialista. Rio de Janeiro: Revinter; 2005.

2. Brasil. Ministério da Saúde. Secretaria de Assistência à Saúde. Instituto Nacional de Câncer. Câncer no Brasil: dados dos registros de base populacional. Rio de Janeiro: INCA; 2003.

3. Eksteen EC, Rieger J, Nesbitt M, Seikalv H. Comparison of voice characteristics following three different methods of treatment for laryngeal cancer. J Otolaryngol. 2003; 32(4):250-3.

4. Carrara-de EA, Furia CLB, Mourão LF, Kowalski LP. Atuação da fonoaudiologia no câncer de cabeça e pescoço. São Paulo: Lovise; 2000.

5. Dedivitis RA, França CM, Mafra ACB, Guimarães FT, Guimarães AV. Características clínicoepidemiológicas no carcinoma espinocelular de boca e faringe. Rev Bras Otorrinolaringol. 2004; 1(70):35-40.

6. Alho OP, Hannula K, Luokkala A, Teppo H, Koivunen $\mathrm{P}$, Kantola S. Differential prognostic impact of comorbidity in head and neck cancer. Head Neck. 2007; 29(10): 913-8.

7. Hendry JH, Branislay J, Zubizarreta EH. Normal tissue complications after radiation therapy. Rev Panam Salud Publica. 2006; 20(2/3):151-60.

8. Brasil. Ministério da Saúde. Instituto Nacional de Câncer. Carcinoma epidermóide de cabeça e pescoço. Condutas do INCA/MS. Rev Bras Cancerol. 2001; 47(4):361-76.

9. Almeida FCS, Cazal C, Durazzio MD, Ferraz AR, Silva DP. Radioterapia em cabeça e pescoço: efeitos colaterais agudos e crônicos bucais. Rev Bras Patol Oral. 2004; 3(2):62-9.

10. Prepageran N, Raman R. Delayed complication of radiotherapy: laryngeal fibrosis and bilateral vocal cord immobility. Med J Malaysia. 2005; 60(3):377-8. 11. Tschudi D, Steckli S, Schmid S. Quality of life after different treatment modalities for carcinoma of the oropharynx. Laryngoscope. 2003; 113:1949-54. 12. Caminero CMJ, Señaris GB, López LA, Núñez BF, Alonso PR, Suárez NC. Voice quality assessment after laryngeal cancer radiotherapeutic

DOI: 10.1590/S1516-18462010005000038

RECEBIDO EM: 28/05/2009

ACEITO EM: 03/02/2010

Endereço para correspondência:

Renata Jacob Daniel Salomão de Campos

Av. Olegário Maciel, 1685/307

Juiz de Fora - MG

CEP: 36016-011.

E-mail: renatajdaniel@ hotmail.com treatment at initial stages. Clin Transl Oncol. 2006; 8(4):284-9.

13. Dubois MD, Crevier-Buchman L, Martin C, Prades JM. Epidermoid carcinoma of piriform sinus after chemo-radiotherapy: acoustic evaluation and voice handicap. Rev Laryngol Otol Rhinol. 2006; 127(5):299-304.

14. Vickery LE, Latchford G, Hewison J, Bellew M, Feber $T$. The impact of head and neck cancer and facial disfigurement on the quality of life of patients and their partners. Head Neck. 2003; 25(4):289-96.

15. Behlau, M, Santos, LMA, Oliveira, G.. CrossCultural Adaptation and Validation of the Voice Handicap Index Into Brazilian Portuguese. J Voice. No prelo 2009

16.Jacobson BH, Johson A, Grywalski C, Silbergleit A, Jacobson G, Benninger MS. The voice Handiscap Index (VHI); Development and validation. Am J Speech Lang Pathol 1997; 6:66-9.

17. Jotz GP, Dornelles S. Auto-avaliação da voz: voice handicap index. Arq Med Canoas. 2000; 3:43-50.

18. Vartanian JG, Carvalho AL, Yueh B, Furia CLB, Toyota J, Mcdowell JA et al. Brazilian-portuguese validation of the University of Washington quality of life questionnaire for patients with head and neck cancer. Published online 5 July 2006 in Wiley InterScience. Disponível em: http://www. interscience.wiley.com. Acesso em 02 de novembro de 2007.

19. Van Goch CD, Verdonck-De Leeuvw M, BoonKamma BA, Rinkel RN, De Bruin MD, Langendijk $\mathrm{JA}$, et al. The efficacy of voice therapy in patients after treatment for early glottic carcinoma. Cancer. 2006; 106(1):95-105.

20. Peeters AJ, Van Gogh CD, Goor KM, Verdonck-De Leeuw IM, Langendijk JA, Mahieu HF. Health status and voice outcome after treatment for T1a glottic carcinoma. Eur Arch Otorhinolaryngol. 2004; 261(10):534-40.

21. Olthoff A, Steuer-Vogt MK, Licht K, Sauer-Goenen M, Werner C, Ambrosch P. Quality of life after treatment for laryngeal carcinomas. J Otorhinolaryngol - Relate Spec. 2006; 68(5):253-8.

22. Goldstein DP, Hynds KL, Christensen AJ, Funk GF. Health-related quality of life profiles based on survivorship status for head and neck cancer patients. Head Neck. 2007; 29(3):221-9.

23. Verdonck-De Leeuw IM, Eerenstein SE, Van Der Linden MH, Kuik DJ, De Bree R, Leemans CR. Distress in spouses and patients after treatment for head and neck cancer. Laryngoscope. 2007;117(2):238-41. 